http://jmscr.igmpublication.org/home/ ISSN (e)-2347-176x ISSN (p) 2455-0450 crossref DOI: https://dx.doi.org/10.18535/jmscr/v10i1.20

\author{
Journal Of Medical Science And Clinical Research \\ IGM Publication \\ An Official Publication of IGM Publication
}

\title{
Original Article \\ Determinants of Publication Pressure and Motivations and Barriers to Publishing among Biomedical Faculty Members
}

\author{
Authors \\ Dr Saeed Mohammed Al-Qahtani ${ }^{{ }^{*}}$, Bayan S Alqahtany ${ }^{2}$, Razan A Al Ghassab ${ }^{3}$ \\ ${ }^{1}$ Assistant Professor KSAU-HS (JA), Consultant, KAMC-Riyadh, Flight Surgeon, Aviation Medicine \\ Department \\ ${ }^{2}$ Senior Resident Family Medicine- King Abdul Aziz Medical City- National Guard Riyadh, Saudi Arabia \\ ${ }^{3}$ Senior Resident Family Medicine- King Abdul Aziz Medical City- National Guard Riyadh, Saudi Arabia \\ *Corresponding Author \\ Dr Saeed Mohammed Al-Qahtani \\ Assistant Professor, King Saud bin Abdulaziz University for Health Sciences (KSAU-HS)
}

\begin{abstract}
Objective: To assess the extent and explore the determinants of publication pressure among faculty members in health sciences colleges

Methods: A cross-sectional study involved 84 faculty members including colleges of medicine, dentistry, pharmacy, nursing, public health and health informatics, science and health professions and applied medical sciences. The Publication Pressure Questionnaire (PPQ-14 items) was used with a calculation of $0-100$ scaled PPQ score (PPQS). High publication pressure was defined as PPQS score $>50\left(75^{\text {th }}\right.$ centile) of the study population. Barriers to publication were assessed using a 10-item scale with calculation of a barrier to publication score (BPS, range=0-40). Stepwise linear regression and binary logistic regression models were used to analyze the independent factors for PPQS and high publication pressure, respectively. Results: The mean (SD) PPQS score was 41.56 (12.46). The prevalence of high publication pressure was $27.4 \%$ (95\% CI=18.2\%-38.2\%). Both PPQS and high publication pressure were independently associated with BPS, as demonstrated in stepwise linear $(B=0.56 ; 95 \% C I=0.15,0.98 ; p=0.008)$ and binary logistic $(O R=1.28 ; 95 \% C I=1.04,1.56 ; p=0.018)$ regression models, respectively. $P P Q S$ was also independently associated with the number of publications in an inversed relationship (B=-3.78; 95\% CI=-5.59, -1.96 ; $p<0.001)$. Having "career progression", "financial incentives", or "awards and prestige" as the first motive for publication was independently associated with PPQS (B=6.73; 95\% CI=0.97, 12.48; $p=0.0 .23)$. Affiliation to medicine, applied medical sciences, nursing or science \& health professions college was independently associated with high publication pressure $(O R=25.89 ; 95 \% C I=1.18,569.58$; $p=0.039)$.

Conclusion: More than a quarter of medical faculty members experience high levels of publication pressure, which is associated with several obstacles to publications and career and financial expectations. Supportive measures should be implemented to enhance research productivity and facilitate the publishing process in the medical academia.

Keywords: Publication pressure, questionnaire, faculty, research, research and publication, scientific publication, academic position, faculty, publication pressure.
\end{abstract}




\section{Introduction}

Scientific medical research is a necessity rather than an opulence to provide best practice to the patients $^{[1,2]}$. Engagement in scholarly activities may be troublesome for physicians, however, they help to implement the research findings in the clinical practice and transfer knowledge from the lab to the bedside ${ }^{[3]}$. Consequently, peer-reviewed publishing is considered a landmark to the scholarly activities ${ }^{[4]}$, a measure of the analytical skills, and advancement in critical thinking of the clinician $^{[5]}$. Therefore, publishing has become a basic standard for the promotion of clinicians and career advancement ${ }^{[6]}$.

However, publishing has laid odd burden on the clinicians, threatening their excellence in clinical practice and mental health ${ }^{[7]}$. Publication pressure was reported to increase due to competition between researchers and colleagues ${ }^{[8]}$. The prestige of the impact factor of the publishing journal and the number of publications foster competition between researchers ${ }^{[9]}$. While competition can excel the quality of the research outcome, the frustration of rejection and the reliance on the number of publications for academic credit create a stressful environment with excess pressure on the physician ${ }^{[10]}$. Additionally, joining high-level hospitals has strict prerequisites including advanced research published in a high-level journal ${ }^{[5,11]}$.

Consequently, the publication presents a tremendous pressure that would lead to burning out $^{[12]}$ among senior researchers ${ }^{[6]}$. Moreover, the pressure to publish was concluded to impact the quality of research and the credibility of results [13]. In addition, failure to meet the publishing prerequisites of the medical institutes may lead the residents and young researchers to dropout of academia $^{[14]}$.

Several barriers to publications have been observed to increase the publication pressure and abandoning academia. Collaborative research funding is not always available and industry-based funding is skepticized. Clinical research hours are not subsumed in the clinical practice hours of clinicians. Therefore, incorporating research into the standard clinical practice is a challenge. Other factors that hinder research and publishing include lack of research training, supervisors, and workrelated stress ${ }^{[5]}$.

Saudi Arabia has attached particular importance to research and publishing. Research training is assimilated into the resident study modules through monthly journal club meetings and lectures ${ }^{[5,15]}$. Moreover, research is mandatory for the promotion of Saudi clinicians by the Saudi Commission for Health Specialties recommendations ${ }^{[16,17]}$. A recent study reported nearly 43,000 papers published between 2008 and 2013 by 25 Saudi universities highlighting the increasing research publication in Saudi Arabia, where medicine was one of the leading research areas ${ }^{[18]}$. According to Scimago Institutions Rankings, Saudi Arabia occupied $40^{\text {th }}$ position with a cumulative number of approximately 66,000 medical publishing documents between 1996 and 2020 ${ }^{[19]}$. However, several clinical practitioners were found to have no published paper despite their awareness of the inevitability of research ${ }^{[20]}$. The main factors attributed to lack of incorporation in research and publishing in Saudi Arabia were concluded to be the deficiency of research skills and facilities, lack of funds, and workload $^{[1]}$.

Therefore, it is crucial to in-depth investigate barriers and motivations to research work among biomedical faculty members to evaluate the research perceptions and attitudes in Saudi Arabia. Explorations of impact of the publishing pressure will allow improving the involvement in research and educational programs to boost the culture of research among medical residency clinicians.

\section{Rationale and Objectives}

Currently, limited papers tackle the issue of publication pressure particularly among medical academics. This study was designed to evaluate the extent and explore determinants of publication pressure among faculty members in the colleges of medicine, dentistry, pharmacy, nursing, public 
health and health informatics, science and health professions and applied medical sciences in King Saud bin Abdulaziz University for Health sciences (KSAU-HS) - Riyadh.

The following objectives were explored:

- To assess the presence and magnitude of publication pressure among the medical academic staff

- To analyze the correlation of publication pressure with motivations and barriers to publication, as well as the participants' demographic and academic factors

- To explore motivations and barriers to publication among the medical academic staff and the associated demographic and academic factors

\section{Methods}

\section{Design and Setting}

A cross-sectional study was conducted at KSAUHS, Riyadh, Saudi Arabia during May 2020 May 2021. The study protocol was reviewed and ethically approved by the institutional review board of KSAU (RYD-20-419812-36896).

\section{Population}

The study involved all faculty members of the following colleges: medicine, dentistry, pharmacy, nursing, public health and health informatics, science and health professions, and applied medical sciences in KSAU-HS. Both joint- and full-time faculty members were included.

\section{Sampling}

Total number of faculty members in the participating colleges was estimated to be 287 . Sample size was calculated to detect an unknown percentage $(\mathrm{P}=50 \%)$ of participants with high level of publication pressure, with $95 \%$ confidence interval, $80 \%$ statistical power and 0.05 type I error. The target sample size was calculated at $\mathrm{N}=165$. A convenience sampling was used to include all eligible and complete participations.

\section{Tools}

A structured questionnaire was designed comprising four parts. Part one included demographic and academic data such as age, gender, college, job, and years of experience. Part two explored experience and motivations in publishing including number of publications by the participants and the ranking of the motivations to publish among six motives, such as "career progression", "requirement for promotion", etc. Part three explored the barriers to publishing by presenting a predefined list of 10 potential barriers and assessing the level of significance of each barrier using a five-option Likert-type agreement scale $(0=$ strongly disagree, $4=$ strongly agree $)$. Part four explored the level of publication pressure using the 14-item version of the PPQ, which was validated for use in medical science ${ }^{(21)}$. It consists of a 14-item (PPQ1-PPQ14) question-naire exploring three domains with intricate items: PP1: pressure to publish personally experienced by the respondent (nine items); PP2: publication pressure in general terms (seven items); and PP3: publication pressure relating to the scientist's position (four items). Each of the items is a fivelevel Likert-type agreement scale, i.e. from totally disagree to totally agree, scored 0-4 for items with negative statements and 4-0 for those with positive statements, respectively. The average time spent to fill out the questionnaire was between 7-10 minutes.

\section{Scores Calculation}

Barriers to publishing score (BPS, range 0-40) was calculated by adding the scores of 10 items.

Raw overall PPQ score was calculated as the sum of 14 items' scores (range $=0,56)$. Further, a 0-100 scaled overall PPQ score was computed by multiplying the raw score by 100 and dividing by 56.

Each of PP1, PP2 and PP3 scaled scores (range=04) were computed as the sum of the respective items' scores divided by the corresponding number of items, i.e., nine, seven and four, respectively.

\section{Procedure}

The questionnaire was edited for an online survey platform. Deans of the colleges were given the option to either provide the email addresses of 
their academic staff (demonstrators, lecturers, assistants, associates, and professors) or circulate the electronic questionnaire internally using the same link. Where emails were provided, an invitational email was sent to prospective respondents with the survey link. Objectives of the study were explained within the email in neutral terms as "work engagement" and "publication culture" to avoid negative connotation. A reminder was sent two weeks after the original email. In either case, consent to participate in the study was collected by an explicit item at the start of the questionnaire and the link was available for one-month duration.

\section{Statistical Methods}

Data was analyzed using the Statistical Package for Social Sciences (SPSS, IBM). All tests were conducted at a significance level $(\mathrm{p}=0.05)$. Descriptive statistics were performed to present the pattern of answers to different parts of the questionnaire; categorical variables were presented as frequency and percentage, while numerical variables were presented as mean and standard deviation (SD). Internal consistency of different scales used in the study was analyzed by calculation of Cronbach's alpha. Factors associated with publication pressure were analyzed by comparing the mean and variance of scaled PPQ score between different factor's categories using independent t-test for binomial and One-way ANOVA test for multinomial variables. The correlation between PPQ score and BPS was analyzed using linear regression; results are presented as unstandardized regression coefficient (B) with 95\%CI. Stepwise linear regression was used to analyze the independent factors of publication pressure, using scaled PPQ score as the dependent variable. Further, scaled PPQ score was dichotomized into low and high pressure using the $75^{\text {th }}$ centile of the study population, and a multivariate binary logistic regression model was performed to analyze the independent factors of high publication pressure; results are presented as odd ratio with $95 \% \mathrm{CI}$.

\section{Results}

\section{Participants' Characteristics}

Ninety-six individuals were engaged in the questionnaire, of whom 92 replied to the questionnaire and 84 fully completed the survey (response rate $=87.5 \%$ ). Of the 84 participants, the mean (SD) age was 43.35 (9.69) years and 58.3\% were male. Medicine College was predominant with $46.4 \%$ participants, followed by dentistry (13.1\%). The most prevalent job title was assistant professor accounting for $41.7 \%$ of the participants, and the mean (SD) years of experiences of the total sample was $14.23(8.82)$ years. Regarding the number of publications, 6 (7.1\%) declared having no publication, while $46(54.8 \%)$ have already published more than 10 papers (Table 1).

\section{Motivations to Publish}

The top three ranking for motives to publish are depicted in Figure 1. Requirement for promotion was reported to be the first motive by $46.4 \%$ of the participants, while it was reported to be second and third most important motive by $21.4 \%$ and $10.7 \%$, respectively. Contribution to science was reported to be the first, second and third most important motive for $22.6 \%, 14.3 \%$, and $22.6 \%$ of the participants, respectively.

\section{Barriers to Publishing}

High load of clinical work represented the most significant barrier to publish with a mean agreement level of 2.8 out of 4 , followed by academic teaching workload (2.58), and administrative workload (2.55). Of note, financial issues were also significant barriers to publish including lack of financial support by funding bodies (mean agreement=2.48) and absence of financial incentives (2.35) (Figure 2).

\section{Internal Consistency of the Study Scales}

Barriers to publishing scale showed an acceptable level of internal consistency with a Cronbach's alpha $=0.704$. The overall PPQ questionnaire showed a high level of internal consistency with a Cronbach's alpha $=0.800$. The PPQ subscales showed moderate-to-acceptable levels of internal consistency (Cronbach's alpha $=0.557-0.721$ ) (Table 2). 


\section{Levels of Publication Pressure}

The mean (SD) scaled PPQ score was 41.56 (12.46) out of 100. Regarding the three PPQ domains, mean scores ranged between 1.44 for PP3 to 1.81 for PP2 (Table 2). The prevalence of high publication pressure was $27.4 \%$ (95\% CI $=18.2 \%-38.2 \%)$.

\section{Factors associated with publication pressure}

The level of publication pressure was significantly higher in younger age category (25-39 years) and decreased with age $(\mathrm{p}=0.034)$ and years of experience $(\mathrm{p}=0.002)$. Additionally, PPQ score was significantly associated with the job title ( $\mathrm{p}=0.006$ ), as it was lowest among professors (mean, SD PPQ score=32.74, 7.99) and highest among assistant professors (46.33, 13.31). Participants in part-time had higher publication pressure than their counterparts, with mean (SD) PPQ score of 46.52 (14.37) versus 40.01 (11.49), respectively. Of note, the level of publication pressure decreased gradually with the number of publications from 54.17 (7.72) among participants with no publishing experience to 35.93 (7.79) among those who already published more than 20 papers $(\mathrm{p}=0.001)$. There was a significant positive relationship between Overall PPQ score and BPS $(\mathrm{B}=0.84, \mathrm{p}<0.001)$. Further, both colleges and main motives for publication were dichotomized into highly versus lowly demanding, depending on their respective mean PPQ scores using the total population mean (41.56 out of 100) as cutoff. Thus, participants who ranked "career progression", "financial incentives", or "awards and prestige" (highly demanding motives) as their first motive for publication had a significantly higher mean PPQ score by comparison with those who reported other motives as their first motive $(\mathrm{p}=0.003)$ (Table 3).

\section{Independent Factors for Publication Pressure}

Results of the stepwise linear regression and multivariate binary logistic regression are depicted in Table 4 and Table 5, respectively. Both PPQS and high publication pressure were independently associated with BPS, as demonstrated in stepwise linear $(\mathrm{B}=0.56 ; 95 \% \mathrm{CI}=0.15,0.98 ; \mathrm{p}=0.008)$ and binary logistic $(\mathrm{OR}=1.28$; 95\% $\mathrm{CI}=1.04,1.56$; $\mathrm{p}=0.018$ ) regression models, respectively. PPQS was also independently associated with the number of publications in an inversed relationship $(\mathrm{B}=-3.78 ; \quad 95 \% \mathrm{CI}=-5.59, \quad-1.96 ; \mathrm{p}<0.001)$. Having "career progression", "financial incentives", or "awards and prestige" as the first motive for publication was independently associated with PPQS ( $\mathrm{B}=6.73 ; 95 \% \mathrm{CI}=0.97,12.48 ; \mathrm{p}=0.0 .23)$. Affiliation to highly demanding colleges including medicine, applied medical sciences, nursing or science \&health professions was independently associated with high publication pressure $(\mathrm{OR}=25.89 ; 95 \% \mathrm{CI}=1.18,569.58 ; \mathrm{p}=0.039)$.

\section{Participants' Flow}

A total 96 individuals engaged in the questionnaire, of whom 92 replied to the questionnaire and 84 returned complete participations (response rate $=87.5 \%$ ).

Table 1. Participants' demographic and academic characteristics

\begin{tabular}{lccc}
\hline Parameter & Unit & Mean & SD \\
\hline Age & Years & 43.35 & 9.69 \\
\hline Years of experience & Years & 14.23 & 8.82 \\
\hline Parameter & Category & Frequency & Percentage \\
\hline Gender & Male & 49 & 58.3 \\
& Female & 35 & 41.7 \\
\hline Nationality & Saudi & 63 & 75.0 \\
& Non-Saudi & 21 & 25.0 \\
\hline College & Medicine & 39 & 46.4 \\
& Dentistry & 11 & 13.1 \\
& Pharmacy & 10 & 11.9 \\
& PH \& HI & 8 & 9.5 \\
& Applied Medical Sciences & 3 & 3.6 \\
& Nursing & 9 & 10.7
\end{tabular}




\begin{tabular}{lccc}
\hline Job & Demonstrator & 6 & 7.1 \\
& Lecturer & 13 & 15.5 \\
& Assistant Professor & 35 & 41.7 \\
& Associate Professor & 21 & 25.0 \\
& Professor & 9 & 10.7 \\
\hline Academic appointment & Full-time & 64 & 76.2 \\
& Part-time & 20 & 23.8 \\
\hline No. publications & None & 6 & 7.1 \\
& $1-5$ & 13 & 15.5 \\
& $6-10$ & 19 & 22.6 \\
& $11-20$ & 21 & 25.0 \\
& $>20$ & 25 & 29.8 \\
\hline
\end{tabular}

PH \& HI: Public health and health informatics

Figure 1. Most popular motives for publication among medical academia
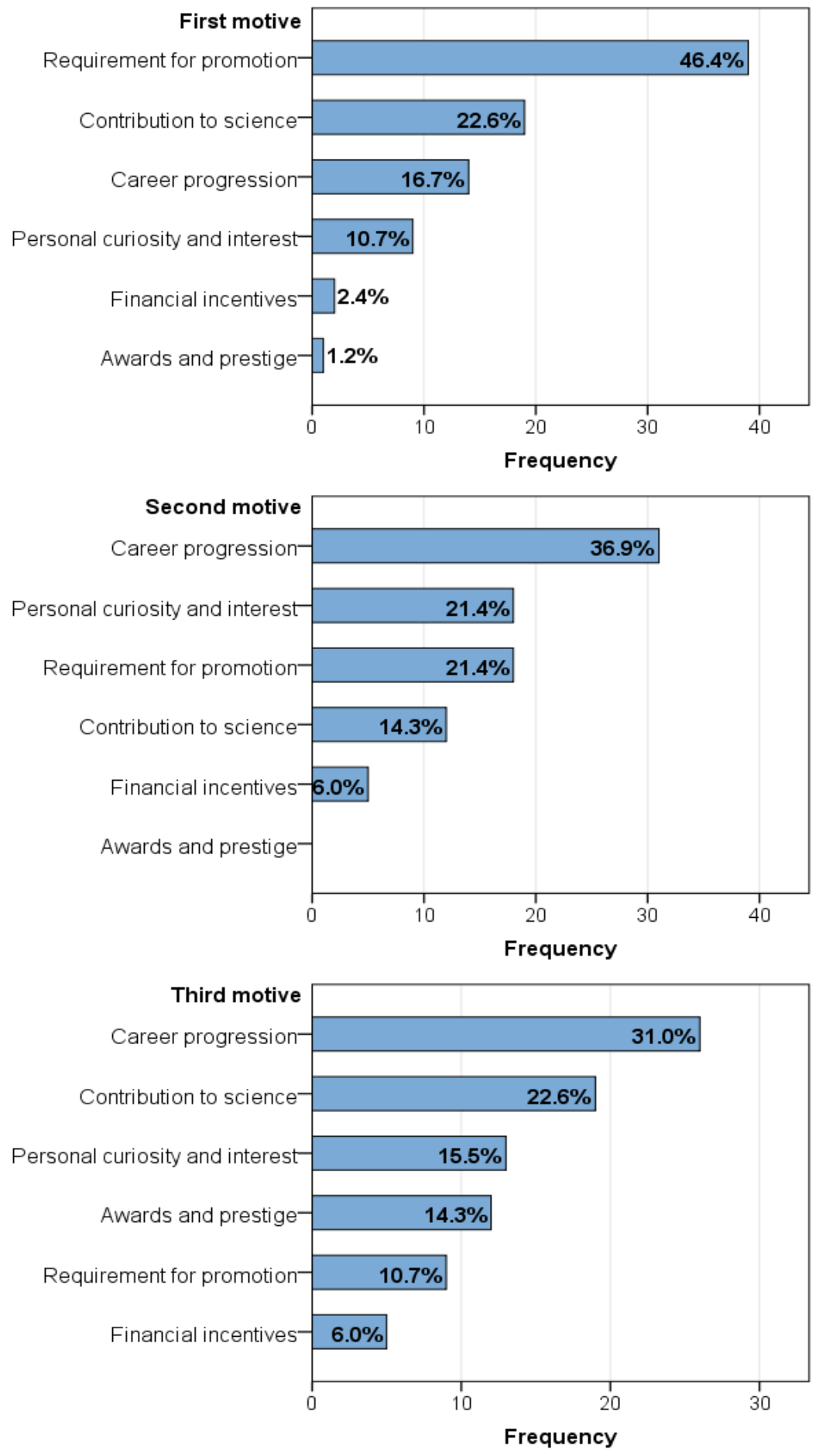
Figure 2. Barriers to publication

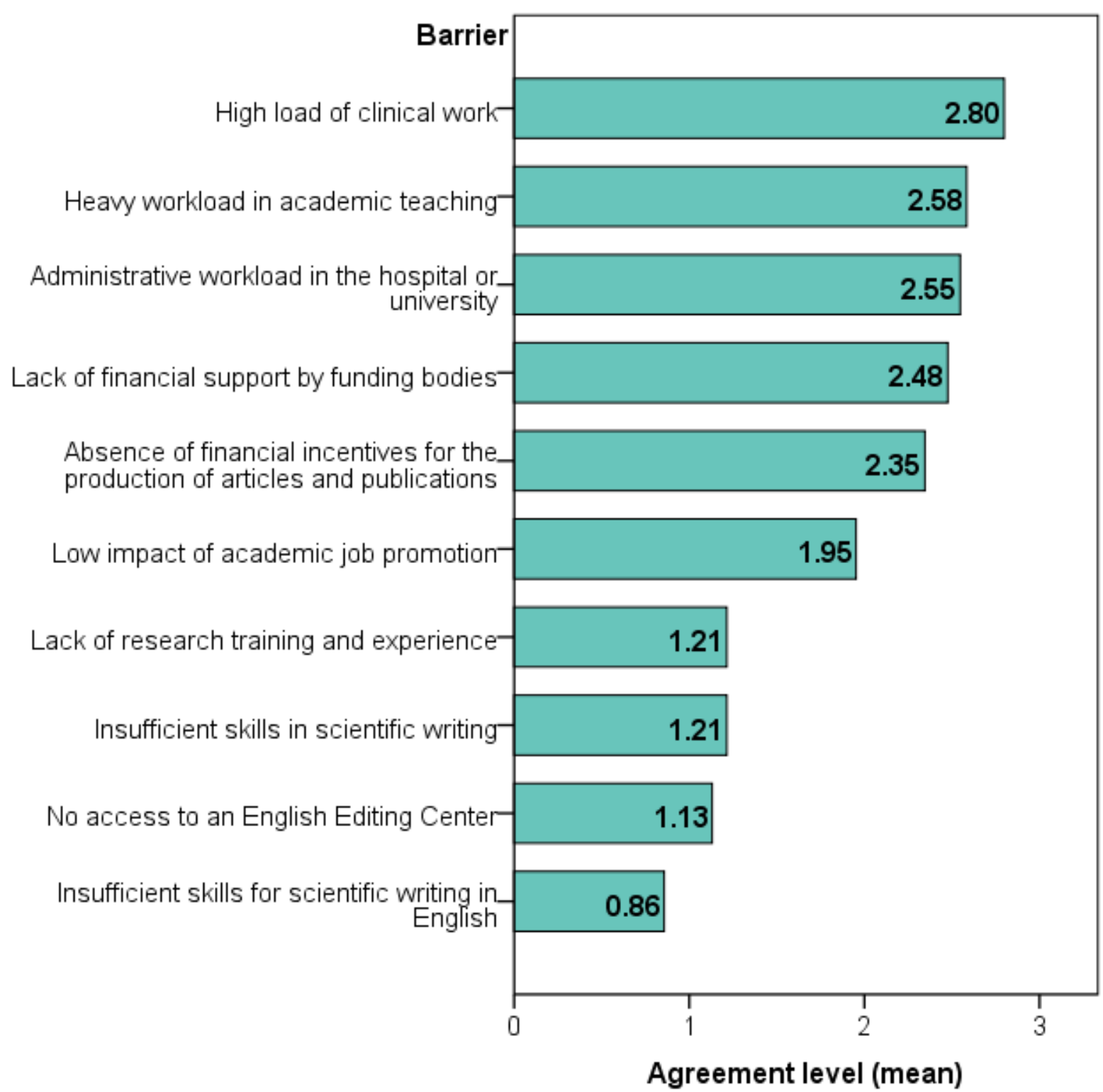

Table 2. Internal consistency of the study scales and descriptive statistics for the respective scores

\begin{tabular}{|l|c|c|c|c|c|c|}
\hline Scale & \multirow{2}{*}{$\begin{array}{c}\text { No. } \\
\text { items }\end{array}$} & $\begin{array}{c}\text { Cronbach's } \\
\text { alpha }\end{array}$ & \multirow{2}{*}{ Reliability level } & \multicolumn{3}{|c|}{ Score statistics } \\
\cline { 5 - 7 } & & & Mean & SD & Range \\
\hline Barriers to publishing & 10 & 0.704 & Acceptable & 19.12 & 5.69 & 8,37 \\
\hline PPQ overall (raw) & 14 & 0.800 & High & 23.27 & 6.98 & 10,46 \\
PPQ overall (scaled) & - & - & - & 41.56 & 12.46 & $17.86,82.14$ \\
\hline PP1 & 9 & 0.691 & Near acceptable & 1.63 & 0.48 & $0.78,3.00$ \\
\hline PP2 & 7 & 0.721 & Acceptable & 1.81 & 0.61 & $0.57,3.57$ \\
\hline PP3 & 4 & 0.557 & Moderate & 1.44 & 0.62 & $0.25,4.00$ \\
\hline
\end{tabular}

PPQ: Publication pressure questionnaire

PPQ1: Pressure to publish personally experienced by the respondent

PPQ2: publication pressure in general terms

PPQ3: publication pressure relating to the scientist's position 
Table 3. Factors associated with publication pressure

\begin{tabular}{|c|c|c|c|c|}
\hline \multirow[t]{2}{*}{ Factors } & \multirow[t]{2}{*}{ Category } & \multicolumn{2}{|c|}{$\begin{array}{c}\text { Level of Publication Pressure (scaled } \\
\text { PPQS) } \\
\end{array}$} & \multirow[t]{2}{*}{ p-value } \\
\hline & & Mean & SD & \\
\hline \multirow{3}{*}{ Age category (years) } & $25-39$ & 45.83 & 15.16 & \\
\hline & $40-49$ & 39.51 & 9.61 & \\
\hline & $50+$ & 37.59 & 9.53 & $.034 *$ \\
\hline \multirow[t]{2}{*}{ Gender } & Male & 39.47 & 12.30 & \\
\hline & Female & 44.49 & 12.25 & .068 \\
\hline \multirow[t]{3}{*}{ Years of experience } & $0-9$ & 46.79 & 15.23 & \\
\hline & $10-19$ & 41.40 & 10.14 & \\
\hline & $20+$ & 34.35 & 6.91 & $.002 *$ \\
\hline \multirow[t]{2}{*}{ Nationality } & Saudi & 43.08 & 13.41 & \\
\hline & Non-Saudi & 36.99 & 7.60 & $.052^{\S}$ \\
\hline \multirow[t]{7}{*}{ College } & Medicine & 41.58 & 13.37 & \\
\hline & Dentistry & 37.66 & 10.91 & \\
\hline & Pharmacy & 37.50 & 4.69 & \\
\hline & PH \& HI & 41.07 & 13.67 & \\
\hline & Applied Medical Sciences & 52.38 & 10.15 & \\
\hline & Nursing & 43.65 & 15.08 & \\
\hline & Science \& Health Professions & 50.45 & 10.55 & .355 \\
\hline \multirow[t]{2}{*}{ College level } & Highly demanding & 43.15 & 13.42 & \\
\hline & Lowly demanding & 38.55 & 9.94 & .108 \\
\hline \multirow[t]{5}{*}{ Job } & Demonstrator & 43.75 & 18.10 & \\
\hline & Lecturer & 42.58 & 11.17 & \\
\hline & Assistant Professor & 46.33 & 13.31 & \\
\hline & Associate Professor & 36.14 & 7.27 & \\
\hline & Professor & 32.74 & 7.99 & $.006^{*}$ \\
\hline \multirow[t]{2}{*}{ Academic appointment } & Full-time & 40.01 & 11.49 & \\
\hline & Part-time & 46.52 & 14.37 & $.041 *$ \\
\hline \multirow[t]{5}{*}{ No. publications } & None & 54.17 & 7.72 & \\
\hline & $1-5$ & 48.63 & 17.28 & \\
\hline & $6-10$ & 43.70 & 12.56 & \\
\hline & $11-20$ & 38.35 & 10.02 & \\
\hline & $>20$ & 35.93 & 7.79 & $.001 *$ \\
\hline \multirow{6}{*}{$\begin{array}{l}\text { Main motive } \\
\text { publication }\end{array}$} & Career progression & 48.34 & 17.35 & \\
\hline & Requirement for promotion & 39.06 & 10.32 & \\
\hline & Contribution to science & 40.88 & 10.33 & \\
\hline & Personal curiosity and interest & 38.89 & 13.51 & \\
\hline & Financial incentives & 52.68 & 6.31 & \\
\hline & Awards and prestige & 58.93 & - & .078 \\
\hline \multirow{2}{*}{$\begin{array}{ll}\text { Main motive } & \text { for } \\
\text { publication level }^{\ddagger} & \end{array}$} & Highly demanding & 49.47 & 15.97 & \\
\hline & Lowly demanding & 39.55 & 10.64 & $.003 *$ \\
\hline Factor & Unit & B (95\% CI) & $\mathbf{R}^{2}$ & p-value \\
\hline Barrier to publish & BP Score & $0.84(0.39,1.28)$ & 0.146 & $<.001 *$ \\
\hline
\end{tabular}

PH \& HI: Public health and health informatics

BPS: Barrier to publish score

B: Unstandardized linear regression coefficient

${ }^{\S}$ By assuming inequal variance between the two groups, the p-value is 0.012 .

${ }^{\ddagger}$ Categories were considered as highly demanding if mean PPQS $>41.56$ out of 100 corresponding to the population mean. 
Table 4. Predictors of publication pressure (stepwise linear regression)

\begin{tabular}{lcccc}
\hline Predictor & B & \multicolumn{2}{c}{ 95\% CI } & p-value \\
\hline Variables included & & & & \\
\hline No. publications (ordinal) & -3.78 & -5.59 & -1.96 & $<.001^{*}$ \\
\hline Barrier to publish (numerical [score]) & 0.56 & 0.15 & 0.98 & $.008^{*}$ \\
\hline Main motive for publication level ${ }^{\ddagger}$ (binomial) & 6.73 & 0.97 & 12.48 & $.023^{*}$ \\
\hline Variables excluded & & & & \\
\hline Age category (ordinal) & -0.06 & -0.07 & 0.74 & .550 \\
\hline Years of experience (ordinal) & -0.07 & -0.06 & 0.57 & .569 \\
\hline Job (ordinal) & 0.02 & 0.02 & 0.53 & .892 \\
\hline Academic appointment (binomial) & 0.03 & 0.03 & 0.82 & .798 \\
\hline
\end{tabular}

B: Unstandardized linear regression coefficient

Table 5. Predictors for high publication pressure defined as PPQS $>75^{\text {th }}$ centile (multivariate binary logistic regression)

\begin{tabular}{|c|c|c|c|c|c|}
\hline \multirow{2}{*}{$\begin{array}{l}\text { Predictor } \\
\text { Age category (years) }\end{array}$} & \multirow{2}{*}{$\frac{\text { Level }}{25-39}$} & \multirow{2}{*}{$\begin{array}{l}\text { OR } \\
10.83\end{array}$} & \multicolumn{2}{|c|}{$95 \% \mathrm{CI}$} & \multirow{2}{*}{$\frac{p \text { p-value }}{.256}$} \\
\hline & & & 0.18 & 658.08 & \\
\hline & $40-49$ & 15.13 & 0.37 & 624.71 & .152 \\
\hline & $50+$ & Ref & - & - & .329 \\
\hline \multirow[t]{3}{*}{ Years of experience } & $0-9$ & Ref & - & - & .955 \\
\hline & $10-19$ & 1.47 & 0.12 & 17.43 & .762 \\
\hline & $20+$ & $\mathrm{NC}$ & $\mathrm{NC}$ & NC & .998 \\
\hline \multirow[t]{2}{*}{ College } & Lowly demanding & Ref & & 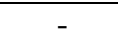 & - \\
\hline & Highly demanding & 25.89 & 1.18 & 569.58 & $.039 *$ \\
\hline \multirow[t]{5}{*}{ Job } & Demonstrator & Ref & 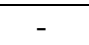 & 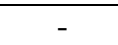 & .615 \\
\hline & Lecturer & 0.11 & 0.00 & 6.92 & 296 \\
\hline & Assistant Professor & 2.72 & 0.10 & 76.82 & .557 \\
\hline & Associate Professor & 0.11 & 0.00 & 705.85 & .624 \\
\hline & Professor & 20.46 & 0.00 & & 1.000 \\
\hline \multirow[t]{2}{*}{ Academic appointment } & Full-time & Ref & - & - & - \\
\hline & Part-time & 0.25 & 0.01 & 5.07 & .364 \\
\hline \multirow[t]{5}{*}{ No. publications } & None & Ref & - & - & .244 \\
\hline & $1-5$ & 4.48 & 0.18 & 109.46 & .358 \\
\hline & $6-10$ & 0.33 & 0.01 & 14.66 & .564 \\
\hline & $11-20$ & 0.07 & 0.00 & 4.13 & 197 \\
\hline & $>20$ & 0.18 & 0.00 & 884.48 & 696 \\
\hline \multirow{2}{*}{$\begin{array}{ll}\text { Main motive } & \text { for } \\
\text { publication level }\end{array}$} & Lowly demanding & Ref & - & - & - \\
\hline & Highly demanding & 12.89 & 0.83 & 201.39 & .068 \\
\hline Barrier to publish & BP Score & 1.28 & 1.04 & 1.56 & $.018^{*}$ \\
\hline
\end{tabular}

OR: Odd ratio; 95\%CI: 95\% confidence interval

REF: category used as reference in the logistic regression equation

*Statistically significant result $(\mathrm{p}<0.05)$

\section{Discussion}

The present study analyzed the factors and determinants of publication pressure among the medical academia. This study determined the motivations and barriers to publication and their contribution in publication pressure. Publishing as a prerequisite of promotion came on top of the list of publishing motivations, followed by contribution to knowledge. This study also revealed that workload whether clinical, academic teaching, or administrative was the most hindering barrier to publishing. Finance stood in second place. The PPQ questionnaire showed a high level of internal consistency, and the prevalence of high publication pressure was estimated as $27.4 \%$. Publication pressure was higher among the young age participants than the elderly. On the other hand, participants with high job titles or high number of published articles experienced less pressure of publications. Further, time availability contributed to the publishing pressure among parttime respondents. This study highlighted the 
motives that confer a high publication pressure to the participants including career progression, financial incentives, and award-and-prestige. Moreover, affiliation to certain colleges such as medicine, applied medical sciences, nursing or science and health professions was associated with a high publishing pressure.

\section{Context of the Study}

The respondents to the PPQ were 84 academic personnel, mostly males, with a mean age of 43.35 years. Majority of the responders were from medicine and dentistry professionals. The job title 'assistant professors' with extended years of experience showed highest response compared to the other job titles. More than half of the respondents have published more than 10 papers throughout their careers.

Estimations of the publication pressure levels in correlation with academic factors and motives to publishing reflect that publishing pressure is mostly experienced at early career stages, notably in colleges where publication is a prerequisite for academic credit and promotion. Afterwards, publication pressure diminishes at career-end, especially after achieving a good number of publications.

These observations support that the demand for publishing exerts considerable pressure on the medical academia and the fulfillment of the demands releases the exerted pressure. Afterwards, research and publishing activities are driven by the researcher's willingness to contribute in knowledge. This was demonstrated by the significantly low PPQ scores associated with having contribution to knowledge as the major motive for publishing.

It is obvious that research activity and publishing are time- and resource-consuming. This explains that unavailability of time for research due to the involvement in the mainstream work duties (clinical practice, educational duties, administrative obligations) increases the feeling of pressure due to the demands of publishing. Similarly, lack of financial support was reported to be one of the major barriers to publication. These two issues of time and finance may be considered in a future institutional or national plan for scientific research promotion.

\section{Agreement and disagreement with other studies}

\section{Motive to Publish}

A study conducted on a random sample of the American Society of Radiologists concluded that personal satisfaction and gratification were the topmost motivations for publishing among the participants $^{[22]}$. A recent study conducted in Netherland proposed that position promotion and career advancement were the most pressing persuasion to publishing ${ }^{[8]}$. Another recent study conducted on medical and nursing educators of different specialties showed that collaboration was a motivating factor for the participants to engage in scholarly activities ${ }^{[23]}$. Career progression and adding to knowledge were the topmost motives for post-graduate dental teachers from various dental colleges in South India, prestige was also included as an important motive for publishing ${ }^{\text {[24] }}$. Our study agrees with the literature as career promotion and contribution to science were the important motives to conduct research and publishing. However, other motives have been highlighted in the literature including personal motives, which may correspond to personal interest that ranked fourth among top motives in our study. Otherwise, the differences in motives between the studies and participants may mark differences in the approach to research between the faculty members; thus highlighting the importance of developing the culture of research at the institutional level, notably among undergraduates and juniors. The current study agreed with the literature ${ }^{[24]}$ that prestige cannot be ignored as a motive for publishing.

\section{Barriers to Publication}

A study conducted in Riyadh Military Hospital primary care centers, Saudi Arabia, reported that insufficient time (due to workload duties) was the 
most frequently cited barrier $(84 \%)$ for conducting research and, hence publishing followed by lack of support (59\%); financial support came at the tail of the list $(38 \%)^{[20]}$. Another study conducted in multiple training centers in the Eastern Province of Saudi Arabia concluded that lack of research skills and facilities for research precedes time availability and funding ${ }^{[1]}$. A recent study conducted in the USA concurred that time availability was the most experienced barrier to publishing ${ }^{[22]}$. Insufficient time received the highest mean in a study carried out on faculty members of a nursing college in a large governmental university in the Eastern region of Saudi Arabia ${ }^{[25]}$. The results of the current study support the literature agreement that lack of time due to workload is a profound barrier to engagement in research and scholarly activities. On the other hand, where time and financial support may be important to consider, efficient measures should be implemented to mitigate modifiable barriers such as inadequate training for research and low confidence in related skills. Lack of research skills was rated as a low barrier in the present study, as explored using four items. However, such results should consider the features of the study sample composed of $77 \%$ of assistant, associate or full professors; hence, does not reflect the necessity of the early-career academia.

\section{Publication Pressure Questionnaire Score}

PPQ was demonstrated to be a valid and reliable instrument to measure the publication pressure ${ }^{[21]}$. Moreover, PPQ was shown to be correlated with burnout and emotional exhaustion, indicators of publication pressure. Also, the cumulative PPQS showed a highly significant inverse correlation with depersonalization and personal accomplishment ${ }^{[12]}$. This study found that the prevalence of publication pressure was $27.4 \%$ and PPQ was correlated with the barriers to publication score.

\section{Factors associated with publication pressure}

Our results are aligned with the literature with respect to the age. Younger the age of the faculty member, more the motives for publishing, and more the pressure experienced. Locally, data from a study involving faculty members in medical and health colleges in Saudi Arabia concurred that least experience had higher percentage of scholarly activities and publishing ${ }^{[26]}$. Contrariwise, a recent study conducted in the four academic universities (including medical schools) in Amsterdam, Netherlands reported that assistant professors perceive a higher level of publication stress than younger Ph.D. candidates, associates, and full professors ${ }^{[10]}$. Data from Nigeria and Beirut emphasized that junior staff member are more likely to engage in scholarly activities and experience higher levels of publishing pressure than the senior $\operatorname{staff}^{[27,28]}$. On the other hand, assistant professors underwent higher levels of publication pressure than assistant or full professors. This can be explained on the basis that the assistant professors are in the midway as they aim for a tenured position (career promotion) that is based on the quantity of publishing. Further, the lowest pressure was observed in full professors may be the hallmark of decline in scholarly activity.

Our study is aligned with the literature that lower number of publications and greater workload were independent factors associated with publication pressure. In a recent study conducted in a large governmental hospital in the Eastern province of Saudi Arabia, the number of publications was the only variable that was concluded to correlate inversely with publication barriers parameters, including personal and organizational barriers ${ }^{[25]}$. Moreover, the workload was associated with a lower percentage of research productivity especially the administrative workload ${ }^{[26]}$.

\section{Publication and Academic and University Requirements}

Requirements set for publishing by universities and colleges reflects the institutional engagement 
to promote publishing and high-quality research, which results in enhancing the faculty performance metrics, and thereby, prestige ${ }^{[29]}$. Universities may encourage publishing by establishing incentives allowance system and academic promotion, thus casting positive pressure on the staff members to publish $^{[30]}$. Moreover, publishing is increasingly demanded for the awarding of academic degrees by universities $^{[29]}$. These observations suggest the importance of designing and implementing a wise strategy that balances the level of scholarly requirement by higher universities to empower engagement in research, without inducing an adverse publishing pressure that may have a negative impact on the academia engagement in research or the quality of the research. In the context of the present study, further research is warranted to understand the determinants of research and publishing culture among specific subgroups of the medical academia. This would enable designing the efficient approach to promote research while fostering a healthy academic environment.

\section{Limitations and Strengths}

One of the strengths of this study is the evaluation of the reliability of the PPQ in medical academia. To the best of the author's knowledge, the evaluation of validity and reliability of PPQ in medical academia were addressed in only a few studies $^{[12,21]}$. Moreover, the study estimated the prevalence of high publication pressure in the participating center and established the significant correlation of the level of publication pressure with the intrinsic and extrinsic barriers to publication. Furthermore, several factors have been highlighted to be associated with publication pressure, where time availability and not the monetary issues came on the top of the list.

However, some limitations hinder the generalizability of the present study findings. Although the response rate was high, nearly $87 \%$, total number of respondents was low, which impacted the power of inferential statistics and thus the internal validity of results. Additionally, non response bias may have arisen given the skewness of the population characteristics notably regarding the academic degree, which in addition to the single-center recruitment impacts the external validity of the findings. Another limitation inherited in the study is that questionnaire is a self-reported tool with subjective items, which induce further bias.

\section{Implications of the Study in Practice}

The current study emphasizes the impact of timespace as a determinant factor of the development of a healthy environment for scholarly activity including research and publishing. Regulatory measures should be considered to allow timespace for academic candidate dedication to publishing. Moreover, measures should be considered by the university authority to promote research culture and implement enablers to enhance scholarly skills, create quality research opportunities, and ease the publishing pressure to the healthy level. Ultimately, the enhancement plan of the university performance metrics should follow reasonable milestones that should consider specific characteristics and requirements of the academia.

\section{Implications of the Study in Research}

The present study recommends conducting a national survey including representative samples of the medical academia from the major biomedical colleges. Such study should explore the research and publishing culture and determine the determinants and drivers for competitive scholarly activity, considering both regional and international scales.

\section{Conclusion}

Publishing exerts a tremendous burden in the early-stage career medical staff, owing to the fact that publication is a prerequisite for academic credit and promotion. The burden of academic requirements obscures the scientific and personal motivation for research, which may have deleterious impact on the scholarly environment 
and research culture both at the individual and institutional levels. Additionally, workload generated by clinical practice, academic teaching, and administrative tasks constitutes a prominent barrier to publishing, followed by lack of financial support. Decision makers should consider addressing the specific characteristics and requirements of the medical academia, which would enable evidenced-based approach in promoting an institutional environment that fosters an internationally competitive scholarly activity. The potential adverse effects of publishing pressure on the academia engagement in quality research not only impact the individuals' careers but also determine the university performance metrics.

The institutional review board of King Abdullah International Medical Research Center KAIMRC (RYD-20-419812-36896).

Self-funded research, no conflict of interest All authors contributed in all steps of the research from discussing the idea to the manuscript writing. The manuscript was confirmed and approved by all authors

\section{References}

1. Saud AlEnazi A, Alamri AS, AlGhamdi AS, et al. Perceptions, barriers, and attitudes toward research among intraining physicians in Saudi Arabia: A multicenter survey. Sci Prog. 2021;104:368504211010604. 10.1177/00368504211010604

2. Chen S, Pan Y, Yao Q, Yao L, Liu Z, Xiang L. Publication pressure on Chinese doctors--another view. Lancet. 2014;384:956.10.1016/S01406736(14)61630-5

3. Duvivier R, Crocker-Buqué T, Stull MJ. Young doctors and the pressure of publication. Lancet. 2013; 381:e10.10.1016/S0140-6736(13)60932-0

4. Bechtold JE, Williams BR, Weinstein SL, et al. The pursuit of scholarship: why we should care about resident research. J Bone Joint Surg Am. 2017;99:e119.10.2106/JBJS.16.01502

5. Al-Mohrej OA, Alsadoun NF, Alshaalan FN, Alturki RI, Al-Rabiah AM, Hamdi NB, et al. Research activities and critical appraisal skills among Saudi orthopedic residents. BMC Med Educ. 2021;21:311. 10.1186/s12909-021-02772-y

6. Laker C, Cella M, Callard F, Wykes T. The impact of ward climate on staff perceptions of barriers to research-driven service changes on mental health wards: A cross-sectional study. J Psychiatr Ment Health Nurs. 2020;27:28195.10.1111/jpm.12577

7. Singh C, Jackson D, Munro I, Cross W. Work experiences of Nurse Academics: A qualitative study. Nurse Educ Today. 2021;106:105038.

10.1016/j.nedt.2021.105038

8. Haven TL, Goede MEE de, Tijdink JK, Oort FJ. Personally perceived publication pressure: revising the Publication Pressure Questionnaire (PPQ) by using work stress models. Res Integr Peer Rev. 2019;4:7.10.1186/s41073-019-0066-6

9. Anderson MS, Ronning EA, De Vries R, Martinson BC. The perverse effects of competition on scientists' work and relationships. Sci Eng Ethics. 2007;13:437-61.10.1007/s11948-0079042-5

10. Haven TL, Bouter LM, Smulders YM, Tijdink JK. Perceived publication pressure in Amsterdam: Survey of all disciplinary fields and academic ranks. PLoS One. 2019;14:e0217931.10.1371/journal.pone.0 217931.

11. Park B, Sohn E, Kim S. Does the pressure to fill journal quotas bias evaluation?: Evidence from publication delays and rejection rates. PLoS One. 2020;15:e0236927.10.1371/journal.pone.0 236927 
12. Tijdink JK, Vergouwen AC, Smulders YM. Publication pressure and burn out among Dutch medical professors: A nationwide survey. PloS one. 2013;8(9):e73381.10.1371/journal.pone.02 36927

13. Fanelli D, Costas R, Larivière V. Misconduct policies, academic culture and career stage, not gender or pressures to publish, affect scientific integrity. PLoS One.

2015;10:e0127556.10.1371/journal.pone.0 127556

14. Levecque K, Anseel F, De Beuckelaer A, Van der Heyden J, Gisle L. Work organization and mental health problems in $\mathrm{PhD}$ students. Research Policy. 2017;46:868-

79.10.1016/j.respol.2017.02.008

15. Mittwede PN, Morales-Restrepo A, Fourman MS, et al. Research-track residency programs in orthopaedic surgery: asurvey of program directors and recent graduates. J Bone Joint Surg Am. 2019;101:1420-7.10.2106/JBJS.18.00472

16. Alsuhaibani M, Alharbi A, Inam SNB, Alamro A, Saqr M. Research education in an undergraduate curriculum: Students perspective. Int J Health Sci. 2019;13:30. https://www.ncbi.nlm.nih.gov/labs/pmc/art icles/PMC6436448/

17. Abdo A, Bodrick M. The commission in 2020: A transformation journey worth taking! Journal of Health Specialties. 2017;5:127-

128.https://www.semanticscholar.org/pape $\mathrm{r} /$ The-commission-in-2020\%3A-Atransformation-journey-Abdo-

Bodrick/74cc4988f9ed5b37ee56ec0f0eab2 f1e1d2244cd

18. Al-Mutairi KA, Al-Shami SA. Scientific research in Saudi universities: science thrives in the desert. GJBAHS. 2015;4:6. https://www.longdom.org/articles/scientifi c-research-in-saudi-universities-sciencethrives-in-the-desert.pdf

19. International Science Ranking. SJR International Science Ranking. (2021) accessed: October 29, 2021: https://www.scimagojr.com/countryrank.p hp? area $=2700$

20. Al-Abdullateef SH. A survey of the attitude and practice of research among doctors in Riyadh military hospital primary care centers, Saudi Arabia. J Family Community Med. 2012;19:38-42. 10.4103/2230-8229.94012

21. Tijdink JK, Smulders YM, Vergouwen ACM, de Vet HCW, Knol DL. The assessment of publication pressure in medical science; validity and reliability of a Publication Pressure Questionnaire (PPQ). Qual Life Res. 2014;23:205562.10.1007/s11136-014-0643-6

22. Clark KR, Jackowski MB. Medical imaging and radiation therapy professionals' perceptions of publishing in academic journals. Radiol Technol. 2020;91:333-41.

https://pubmed.ncbi.nlm.nih.gov/3210286 $1 /$

23. Clark KR, Webster TL. Scholarly productivity among educators in radiologic sciences and other health care professions: a comparative approach. Radiol Technol. 2020;92:113-25.

https://pubmed.ncbi.nlm.nih.gov/3320376 $8 /$

24. Srinivasan MR, Poorni S, Sujatha G, Kumar SN. Research experiences, attitudes, and barriers to publishing among the dental postgraduate teachers: a crosssectional study. Indian J Dent Res. 2014;25:454-8.10.4103/09709290.142529

25. Darawad MW, Alhussami M, Sa'aleek MA, Al Ateeq E, Samarkandi OA, AlAnati A. Nursing faculty members' attitudes and perceived barriers toward 
conducting scientific research: A descriptive study from Saudi Arabia. Int J Caring Sci. 2018;11:1192-203. http://www.internationaljournalofcaringsci ences.org/docs/63._1-1darawad_original10_2.pdf

26. Alghanim SA, Alhamali RM. Research productivity among faculty members at medical and health schools in Saudi Arabia. Prevalence, obstacles, and associated factors. Saudi Med J. 2011;32:1297-

303.https://pubmed.ncbi.nlm.nih.gov/2215 9387/

27. Lesi OA, Orenuga OO, Roberts A, Abudu OO. Research productivity of junior academic staff at a tertiary medical college in south west, Nigeria. Nig Q J Hosp Med. 2009;19:119-

24.https://pubmed.ncbi.nlm.nih.gov/20836 313/

28. Dakik HA, Kaidbey H, Sabra R. Research productivity of the medical faculty at the American University of Beirut. Postgrad Med J. 2006;82:4624.10.1136/pgmj.2005.042713

29. Mills D, Inouye K. Problematizing 'predatory publishing': A systematic review of factors shaping publishing motives, decisions, and experiences. Learned Publishing. 2021;34:89104.10.1002/leap. 1325

30. Demir SB. Predatory journals: Who publishes in them and why? Journal of Informetrics. 2018;12:1296311.10.1016/j.joi.2018.10.008. 